\title{
Nursing Home Residents' Perceptions on Ego-Integrity Achievement in Nursing Home
}

\author{
Hyesun Park ${ }^{1}$, Sunyoung Lim $^{2}$ \\ ${ }^{1}$ Ph. D, 06974 Dept. of Red Cross College of Nursing, Chung-Ang University, 221 Heukseok-dong, \\ Dongjak-gu, Seoul, Republic of Korea, april1007@naver.com \\ ${ }^{2}$ Visiting Professor, 31065 Dept. of Nursing, Baekseok Culture University, 1 Baekseokdaehak-ro, \\ Dongnam-gu, Cheonan-si, Chungcheongnam-do, Republic of Korea, isokety@naver.com
}

Corresponding author: Sunyoung Lim

\begin{abstract}
This study was to uncover the nature of in-depth and specific experiences by identifying the awareness of the achievement of ego-integrity among senior citizens living in nursing homes. The purpose of this is to provide basic information on the achievement of ego-integrity of the elderly living in nursing homes. The subjects were 7 elderly people living in two elderly care facilities in Gyeonggi and Incheon, Korea, with whom one-on-one in-depth interviews were conducted. The data were collected from December 2019 to January 2020 and analyzed using traditional content analysis. Content analysis is a systematic and objective method that analyzes explicit or potential content by classifying and evaluating it to confirm the meaning and effect of major data symbols or subjects. Upon the analysis, the nursing home residents' perceptions revealed five categories of means to achieve ego integrity: maintaining harmonious relationships, a companion to talk with service, maintaining autonomous functions, maintaining passive and active activities, and spiritual stability from participating in religious activities. The results of this study are meaningful in that they presented the necessary documents and supporting data to prepare a management plan for the nursing practitioners for the achievement of ego-integrity of the elderly living in nursing homes. Based on this study's results, follow-up research is necessary to verify the effectiveness and development of nursing practitioner education and intervention programs to help nursing home residents achieve ego integrity.
\end{abstract}

Keywords: Nursing Home Residents, Ego-Integrity, Perception, Content Analysis

\section{Introduction}

As the world's aging population has grown and continues to grow, many countries have adopted long-term accommodation care as a major welfare policy, and the number of elderly people receiving care at nursing homes has increased significantly[1]. Some elderly people live in nursing homes due to the aging process. As one age, the rate of developing health conditions that require external observation and care rapidly rises, thus necessitating the continuous prevention of complications and the long-term nursing of chronic diseases. As such, the number of elderly people who require longterm care and suffer from reduced disease resilience also increases, which in turn sees multiple elderly individuals decide to move into nursing homes or similar institutions[2][3]. Moreover, most residents in elderly care centers need help from others to maintain basic daily routines due to their poor physical conditions, or otherwise have complex health problems from various chronic physical, social,

Received: August 06, 2020; $1^{\text {st }}$ Review Result: September 24, 2020; $2^{\text {nd }}$ Review Result: November 12, 2020 Accepted: December 28, 2020 
psychological and mental diseases and therefore require individual nursing[4].

In recent years, successful aging and ego integrity have emerged to emphasize the positive aspects of old age. Erikson first presented a sense of ego integrity as an important development task to achieve in old age, as ego integrity is the result of successfully adapting one's age to the skills and abilities required by society to acquire the developmental tasks that face each stage of life. If successful aging consists of the healthy and active state of old age, a sense of ego integrity, which even encompasses death, is perceived as a sign of lifecycle integration that the elderly can pursue[5][6]. Ego integrity is also considered a general and comprehensive concept that reflects the state of one's psychological well-being and harmonious view of life in the past, present, and future.

As such, the continuous increase of the elderly living in nursing homes suggests that there is a need for measures to maintain psychologically stable health while the elderly live longer.

While elderly people with ego integrity accept death as part of their lives, live as subjects of their own lives, and have gained the wisdom of living with deep consideration for people[7], research has often suggested that elderly individuals lacking ego integrity fear death and often express disgust, pessimism, or chronic contemptuous displeasure[8].

The ego integrity achievement of the elderly is presented as an important area that can improve the quality of life of the elderly in the areas of residual function management and well-dying. In the study conducted by Lim Sun-young et al.[9], the main point of view is the management of residual functions of elderly people living in nursing homes, among which the circumstances of physical, mental and social needs are related to the realization of perceptions of ego integrity achievement. In particular, spiritual demands at nursing homes for the elderly include death-bed nursing, which is managed until the death of the elderly. In nursing, spiritual well-being is an important point of view, and it includes parts that focus on spiritual function until the moment of death to bring about peaceful death, as part of the ego integrity of the elderly[9]. The quality of life in old age and the of ego integrity should be considered comprehensively in the the physical, mental, social and spiritual areas. Because achievement are affected by the need for ego integrity and well-dying and how death is perceived or what meaning is given[7].

Therefore, as the number of elderly people who spend their last days in nursing homes increases and life expectancies at such facilities advances, the nursing practices at nursing homes are important to consider in nursing management. As such, research is needed to explore nursing home residents' perceptions of ego integrity achievement.

\section{Methods}

\subsection{Research Design}

The researchers of this descriptive qualitative study conducted interviews and assessed the interview details via content analysis. Descriptive qualitative research aims to express and comprehensively summarize events to explore the participants' language without applying a specific philosophy or theoretical framework[10]. Accordingly, the main purpose of this study was not to analyze abstracted subjects derived from the data, but rather to summarize and describe the data as they are.

\subsection{Research Subject}

The participants in this study were 7 residents of two nursing elderly care facilities, aged 65 years or older (average age of 78.5 years), whose physical functions were deteriorated but whose cognitive functions were normal. They understood the study's purpose and method and agreed to participate in it. The purposed and snowball sampling methods were used to recruit participants. The residence 
period of the elderly was 27.8 months, and as an elderly with elementary school education, there was no problem in communication. They had hypertension, diabetes, stroke, and heart disease as comorbid diseases [Table 1].

[Table 1] Characteristics of the Respondents

\begin{tabular}{|l|l|l|l|l|}
\hline & Age(years) & Sex & $\begin{array}{l}\text { Length of residence } \\
\text { (months) }\end{array}$ & Comorbid diseases \\
\hline 1 & 84 & Female & 36 & Hypertension \\
\hline 2 & 82 & Male & 26 & Hypertension, diabetes, stroke \\
\hline 3 & 76 & Female & 24 & Heart disease \\
\hline 4 & 71 & Female & 17 & Hypertension, diabetes \\
\hline 5 & 82 & Female & 33 & Hypertension, diabetes \\
\hline 6 & 75 & Male & 28 & Stroke \\
\hline 7 & 80 & Female & 31 & Hypertension, diabetes \\
\hline
\end{tabular}

\subsection{Data Collection Method and Procedure}

The researchers developed questions from a literature review to grasp the nursing home residents' perceptions of ego integrity achievement. The main question was, "Your physical functions are in a difficult state, but please tell me about achieving a sense of ego integrity." More specific questions were, "What do you think can help nursing home residents achieve ego integrity?" and, "What are strategies to promote nursing home residents' ego integrity achievement?"

The data collection period was December 2019 to January 2020, during which two researchers conducted semi-structured individual interviews with the participants. The two researchers had completed qualitative research methodology and qualitative data analysis courses at graduate school and participated in qualitative research conferences and workshops. Each interview took an average of 50 minutes and was recorded with a recording device. The participants' non-verbal expressions, such as non-specific speech, facial expressions, and gestures, were observed and recorded in a field notebook. The participants' consent was confirmed before recording the interviews. To ensure the data's reliability, it was transcribed and saved as an audio file immediately after the interview.

\subsection{Data Analysis}

The data were analyzed through traditional content analysis. Content analysis is a systematic and objective method that analyzes explicit or potential content by classifying and evaluating it to confirm the meaning and effect of major data symbols or subjects[11]. It is a dynamic analysis method that inductively derives results[12]. To start, the researchers attempted to understand the full contents of the interviews by reading the transcripts and field notes several times. Significant phrases related to the nursing home residents' perceptions of ego integrity achievement were identified, displayed, and coded to well represent their inherent meaning and context. After that, the extracted codes were repeatedly categorized and compared according to their meaning to finally answer the research question of what nursing home residents' perceptions of ego integrity achievement are.

\subsection{Ethical Consideration}

The researchers explained to the participants the study's purpose and method, the risks and benefits 
of participating in the interviews, and how to secure confidentiality. The researchers also ensured the participants that they could withdraw their consent at any time during or after the interview if they did not want to continue, and there would be no disadvantages to doing so. Once the participants voluntarily agreed to participate in the study, their written consent was obtained. The collected data were used for research purposes only, and the participants' information was kept strictly confidential. Of the interview contents, personal identification information was replaced with a symbol, and the transcript files were deleted after the transfer was completed. The personal information collected during the research data collection process will be used only for research purposes, the information that can be identified by personal information has been collected coded, and the data collected has been stored in a locked locker, and all research participants' information will be kept for one year after analysis and reporting of research results. The elderly residents who participated in the interview were provided with a gift in return worth 20,000 won.

\section{Results}

Five categories were derived from nursing home residents' perceptions of ego integrity achievement [Table 2].

[Table 2] Essential Category of Ego-Integrity Achievement in Nursing Home Residents

\begin{tabular}{|c|}
\hline Category \\
\hline Maintaining harmonious relationships \\
\hline A companion to talk with service \\
\hline Maintaining autonomous functions \\
\hline Maintaining passive and active activities \\
\hline Spiritual stability from Participation in Religious Activities \\
\hline
\end{tabular}

\subsection{Maintaining Harmonious Relationships}

Maintaining harmonious relationships with surrounding people proved an important part of ego integrity achievement. To the nursing home residents, their fellow residents, nurses, and nursing home staff sympathized with their stories, expressed similar feelings, and supported them, which they noted as helping to achieve ego integrity. In particular, they recognize ego integrity in areas where they show attitude of living in harmony with others by caring or accepting others generously, caring for others while maintaining self-restraint, and caring for the elderly who listen to other people's stories, and thinking about the elderly people who are related to them first:

"When I talk to an old lady, I like it because the process of our lives is so similar. I just feel the rewards of having to send my children to college. And, no big deal, but thank you for keeping my nurse's promise. If you make a promise, even a small promise, keeping it in itself prevents you from feeling depressed and losing self-esteem. Even before I came in, I liked being with people and had a 
lot of friends. Even if I'm here, I like to stay with people in the same situation as me." (Participant 1)

"How nice it is to program with the people here and get along with them. And my stiff body can move, draw pictures and listen to my story. As expected, people have to communicate with people. How much longer would I live if I did? We get along well until the end, and as a good person, that's enough. The director, the nurse, the physical therapist, the restaurant lady, she's so kind and nice." (Participant 3)

"When I'm home alone, I eat alone, eat only about two meals a day, sometimes I'm depressed, and sometimes I don't because I'm sad, but I like to eat, exercise, and do various programs here at the set time. I love talking to other grandmothers, especially nurses talking to talk to them. Especially when I'm not feeling well, it's better to ask for help nearby, but I'm relieved to be able to solve this problem. When I'm alone, I can't ask for help comfortably even if I want to, and it was so touching because I spent a lot of time alone But if I can't get used to it, I tried to go back home, but fortunately, I feel more comfortable than I thought. I can feel that the nurses are taking good care of the grandmothers. I feel at ease. It's also good to ask for what you need. " (Participant 6)

"I love singing here, dancing if I want to, and playing games if I want to. When I was alone, I just turned on the TV and ate alone, and sometimes I ate once a day or not because I was too lazy to eat. I come here and eat with other grandmothers at the same time, give them different side dishes every time, and I don't like all the diets, but I feel relieved just to have a nutritionist and take care of them. I think it's good just to not cook at home. When I'm alone, I can't solve things when they're broken in my house, and I just lived with the inconvenience. But I don't have to worry about it because I have a manager and a manager here, and I'm not worried because I'm trying to solve it if I tell you something that's inconvenient to use. When I lived alone, there were times when I was so tired of the day, and I had no time to laugh. But all the elderly are gathered here, so when they wake up in the morning, they ask each other how they slept, how they feel, and say hello to each other, and support each other. Grandmothers all live in their own style, so sometimes they argue with each other, but maybe because they live a long life, they make up with each other quickly. I understand that. How fortunate is it that nurses and staff here mediate even if there is conflict? I think this is one of the good things. Even in conflict situations, nurses listen to both sides and talk to each other, so misunderstandings are easily solved." (Participant 4)

\subsection{A Companion to Talk with Service}

A companion to talk with about one's interests helped the elderly residents recognize symptoms and naturally accept their current image or situation, thereby contributing to ego integrity. In addition, the data analysis revealed that the nursing home residents felt the companion talk with service helped them recover their cognitive function and thus further achieve ego integrity:

"I need more time to lie down because I am physically stiff now, but I like to use a wheelchair and participate in the program because my mind is healthy. Our nurse said that through the companion to talk with service, cognitive function could be restored further. If you lie on the bed for a long time and your mind is bad, it will be darker. It's also good that the caregiver talks a lot like my daughter, so it feels like talking with my family at home." (Participant 4)

"I lived alone and had a hard time getting used to it since I just got here. Thanks to the grandmothers, nurses, nursing care workers, and social workers on my floor who took a lot of interest in me and talked to me a lot, I was able to adjust quickly. I used to go to the senior citizen center every morning and hang out with my grandmothers because I live alone at home, but I'm glad they're here. "They're busy living, so they call once a month, and they don't have time to talk to me..." (Participant 6)

"I am sad but thankful for the nurse explaining my disease so that I can understand it in detail. 
When I was outside, I didn't try to know about my disease, but just covered it up and tried to pass. Because it's desperate. But when I came here, my mindset changed. Knowing my disease, how can I be cured, and I feel like I want to die more comfortably, so I naturally accept my situation. Now I'm happy that my illness is not hopeless and I can comfortably talk to the nurse about my illness." (Participant 7)

“ I've been taking blood pressure medication since I was in my 60s and now I'm 80 years old, but when I'm alone at home, it's so long and there are many times when I can't remember if I took the medicine. Whenever that happened, I thought about whether to take medicine or not and I stopped taking it. At first, I thought a lot about coming in here and was reluctant to come in, but now that I'm here, there are nurses and I'm so relieved that they take care of my medicine on time. I talk to my nurse while taking medicine. I take good care of my time and compliment me because I take medicine. Even if it's not a big deal, important to take medicine. I'm curious about it. When I ask questions several times, it seems like they understand my feelings easily, so I feel like I'm receiving support. When I lived alone, I suddenly felt lonely and my life was so sad. As I got older, my knees hurt, and whenever I felt my energy decreased, I wanted to live more and die faster. Then I came here, and I'm so thankful that I talked to other grandmothers and that the staff here always asked me about my health and mood. I don't get tired of asking about me and checking how I feel every day. Now I want to spend the rest of my life comfortably here, and I'm so relieved that there's someone who can help me the end. Here, you talk to each other while watching the news, and you explain what I don't know, which solves the frustration a lot. Talking to each other about how the world is going makes me think too. When I was alone, I felt lonely because I had no one to talk to." (Participant 1)

\subsection{Maintaining Autonomous Functions}

Part of maintaining independence are efforts to improve autonomous functions, which encourage the elderly to do what they can and have positive thoughts to promote ego integrity. The participants showed that continuing such simple functions motivates and promotes autonomy and positively influences their independence:

"Our caregivers do not let me fill up diapers every hour just because I'm lying down, but do stool management training so that I don't wear diapers or pads as much as possible, and they meet all my requirements. And I can use a spoon with my right hand without rushing to eat. I can say that I am good at spooning, so I feel good and wait for a mealtime." (Participant 3)

"My director, told me to take your time eating, but no one's going to take care of the plate, so you can use your spoon, your chopsticks, and your fork to eat... When I was outside, my friend went into a place like this and visited me, and I felt gloomy when I saw him eating without even knowing that the food was going into his nose. But they always wait here. [...] so that I could do something even a little. But they always wait here. [...] so that I could do something even a little." (Participant 4)

"When I was alone, I spent a lot of time lying down, sleeping during the day, and sleeping at night. But I felt so good while drawing and making pictures during the day here. I explain what I drew, what I made, and laugh while watching other grandmothers. I should have done this before. Now I realize that I'm also a painter and maker. I am also a person who is colorful and pretty and likes to decorate... I get help when I wash my hair here, but I always brush my hair and apply lotion. I didn't do this when I was alone, but now I'm doing it regularly, and I like to move my hands and take care of myself. I want to do better because if I make a little change, people around me will compliment me. (Participant 2)

"I try to wear even a pair of socks every day. I'm grateful that I can do even a small thing myself. Although it's funny and sad because I'm not good at wearing them, it'd be nice if you compliment me for doing a good. Getting in here means there's a lot you can't do on your own, and as you get older and sick, you just have to get help. But it's lonely to stay still and get help, so I think about and 
practice what I can do by myself, even if it's small. " (Participant 6)

\subsection{Maintaining Passive and Active Activities}

The participants mentioned passive and active activities as a means to combat declining physical functions, as these activities maintain and improve their current muscular strength, which in turn encourages their ego integrity achievement. They also noted that it is important to understand chronic disease control by considering the physical dysfunctions that may accompany health complications:

"If I keep lying in bed like this, my body stiffens. Therefore, the caregiver rolls up a handkerchief and rubs my back. [...] I do physical therapy once in the morning, but it's not all about physical therapy. I have to do it steadily. I change my position, so my body is less stiff, and I change clothes often because I have bedsores, but I don't do it alone and listen as much as I can and lift my buttocks as much as I can. I'm proud to be moving." (Participant 5)

"I can sleep well because I move a lot. When I was at home, I used to take sleeping pills because I couldn't sleep, but here I did physical therapy, and here a nurse came to me to do hand exercises, and the caregiver made me move around a lot, so I started to exercise a lot. That's why I can't help falling asleep." (Participant 2)

"When I was home alone, I often thought that I could live in the same pattern every day and die quickly. But thank you for coming here and walking me around, supporting me, burning my wheelchair to see the sun when I'm having a hard time, explaining about plants, trees, and flowers. I'm so grateful to see you trying to get me up and move and help me when I'm depressed. I feel encouraged and refreshed because of your support. I think I get to work out harder. I feel myself in better shape than when I'm lying down all the time. I'm so happy that people here compliment and encourage me when I do anything by myself. When I'm home alone, it's so hard to wash, put on anything, and didn't do well with cleaning. Everything was pointless, depressing. Some days I slept 15 hours a day without eating. But he's alive when he opens his eyes without dying. Ever since I came here, I've washed my face every day, brushed my teeth, and when I'm having a hard time, a caregiver helps me and always praises me and encourages me when I try to do it myself. It's good to hear compliments. I want to do better than other grandmothers, so I do it more. This is good because I don't have to do the laundry myself. I just want to take care of my health. I feel better now. I hope you stay healthy and healthy. I will try harder." (Participant 4)

"Care worker wants to sit in a wheelchair because it's hard to walk with a cane. Then I don't stay still as soon as I move. Now how you move here because they know very well in hand and leg by a little bit sideways and we can sit down. It's annoying that my body isn't following me well, and it might be harder for my care worker, but I'm proud that I can test myself to see if I can move my body and move my body." (Participant 7)

\subsection{Spiritual Stability from Participation in Religious Activities}

The nursing home residents recognized that spiritual stability resulting from participation in religious activities was a process of accepting and preparing for their death and that of their friends, as well as achieving a sense of ego integrity. It is connected to spiritual well-being that one can have transcendental thoughts at the end of one's life, overcoming anxiety over death and overcoming the development crisis related to death:

"I'm not as highly supported as by religion I love the time to hum hymns here and pray at mealtimes. I feel comfortable when I pause and then say what I want to say. And the pastor comes twice a week. When I hear interesting Bible stories, I'm God. [...] The grandmother across the other side reads the Buddhist scriptures, and it looks fun too. [...] Well...the most important thing I want to 
say is that religion helps me die well. Thank you for giving me a compliment and allowing me to look back on my life for what I did well. Should I say that I have peace of mind? It's good to keep my eyes from being afraid until I close my eyes." (Participant 2)

"Is it sad that my lady is dying? Scared? I'm not sad at all." I used to have a religion, but I'm trying to come here and look back more and pray, repent and die a good death. Doesn't my face look comfortable? My kids come here once a week and they tell me that I look better than when I'm home. I've worked so hard as a mother and a wife, and I've lived without doing anything bad, so it's no better than going to heaven. "And ask them to help you die after living a little less painful life while praying. Then I don't want anything more." (Participant 1)

"When I was young, I was a Sunday school teacher at the church, and from that experience, I love talking about the Bible and talking to each other. Now that it's time to prepare for death, other grandmothers are all sympathizing and expressing their fear and anxiety about death. All we want is to die comfortably. Then there's nothing more I want. My wish is to die in my sleep." (Participant 7)

"Since we're all old people here, there's something in common that everyone thinks about death. I can talk naturally and feel that we all have the same mind. I always pray when I sleep and I have no regrets even if today is the last day. I'm relieved that I'll take care of it even if I die. When I was alone, I was worried about death even if I had a religion. But when I came here, I saw other people, and I knew I wasn't the only one.And now I feel a little at ease about death, and I think I'm ready. It was a short and long life to look back on the past me, but I think I lived hard. I have some time to cheer myself up. I naturally talk about death with other grandmothers. Even if we talk about this, we can relate to each other because we have similar feelings. (Participant 5)

\section{Discussion}

Of the five categories stemming from the nursing home residents' perceptions of ego integrity achievement, both maintaining harmonious relationships and the companion to talk with service approach ego integrity with love rather than nursing skills. The residents also believed empathy for nursing home residents is important. This shows that nursing home residents can identify ego integrity achievement from the way they think, empathize, express and support their feelings, and live together in the same space, just as a family[4][13]. The elderly living in nursing homes have a combination of personal illness and health problems as well as poor physical function, so they can receive individual nursing care[4] and have a low sense of ego-integrity due to fear of death[5]. Therefore, in order to maintain a psychologically stable state of health, it is necessary to help them recognize them as the subject of their lives[7] and to have a high sense of ego-integrity[6].

The elderly living with harmonious relationships can recognize whether emotional ties are good through communication and contact with the nursing home staff who share their daily lives with the elderly even before entering the nursing home. These elderly people have worked hard all their lives to support their families and maintain close relationships, so they have a high level of satisfaction, an emotion that is well adapted and feels satisfied and happy.

The categories of maintaining autonomous functions and maintaining passive and active activities revealed that cognitive functions can decline despite long-term good mental health. However, the study participants told that they were continuously given residual function management implemented under nursing guidelines in nursing homes, and stimulating these cognitive and physical functions could help achieve ego integrity[9]. Lim Sun-young et al.[9] also described the management of nursing home residents' residual functions as the achievement of ego integrity among the residents, as their physical, mental, and social conditions are engaged. This requires sufficient encouragement for the elderly themselves to do it, and giving enough time to the elderly living at meals is necessary for achieving ego integrity. In elderly care centers, most elderly residents are ill-fated elderly who need 
help from others, but it is important to maintain the functional independence of the use of spoons even if they are ill, and it is necessary to provide minimal assistance in the way of meals.

Spiritual stability from participating in religious activities and maintaining harmonious relationships with neighbors is also linked to ego integrity achievement. Specifically, the nursing home residents recognized spiritual stability from participation in religious activities as the achievement of ego integrity, as death-bed nursing requires an important view of spiritual well-being and a peaceful death as part of achieving ego integrity[9][14][15]. This is all the more significant in recent nursing studies because death-bed nursing has become an important aspect in helping nursing home residents accept and prepare for death. Moreover, residents should not just prepare for the moment of death, but also consider living the rest of their lives with happy and healthy minds[16-18]. For elderly individuals living in nursing homes, the focus on physical, cognitive, social, and spiritual functions until the moment of death demonstrates the characteristics of nursing that manage even peaceful deaths [9][14][19][20], which implies a broad and deep meaning. Therefore, in order to achieve ego integrity among the elderly living, the internal resources of spirituality should be utilized, but in order to maximize the effect, the spiritual needs of the elderly residents should be assessed and the contents of social support based on their spiritual needs should be selected, the intervention of nursing for spiritual stability of the elderly living in the residential area. Religious activities and beliefs serve as a buffer against the emotional blow from the loss of greatness in old age, and as death nears, religion at the psychological level helps to face impending death, continues to feel the significance and importance of life, and requires spiritual stability scheming.

\section{Conclusions}

This qualitative study examined elderly individuals living in two nursing homes in Gyeonggi and Incheon, Korea. to understand their perceptions of ego integrity achievement. As a result of this study, the nursing home residents perceived the following to encourage ego integrity achievement: maintaining harmonious relationships, a companion to talk with service, maintaining autonomous functions, maintaining passive and active activities, and spiritual stability from participation in religious activities.

There are some limitations to the results' generalizability. This study has a limitation in that the severity of the elderly living in a nursing home for the elderly is similar, and the difficulty of various disease groups of the elderly is not covered. Since this study did not confirm the difference according to the period of residence in the elderly care facility, it should be studied by improving these limitations in subsequent studies. However, given the increasing importance of managing residents' functions, including ego integrity, in the clinical elderly nursing field, there is a need to prepare a management plan for nursing practitioners to help residents achieve ego integrity. Therefore, it is meaningful that this study has revealed nursing home residents' own perceptions of ego integrity achievement, which can help shape nursing home management plans to help even more residents. For future research, the researchers propose an additional study that evaluates the effectiveness of nursing practitioner education and nursing intervention program development, as based on the revelations demonstrated in the current study, to further aid nursing home residents' ego integrity achievement in the future.

\section{References}

[1] Y. T. Laflamme, M. Tousignant, D. Lussier, P. Lebel, M. Savoie, L. Lalonde, M. Choinière, Educational needs of health care providers working in long-term care facilities with regard to pain managemen, Pain research and 
management, (2012), Vol.17 No.5, pp.341-345.

[2] Garatachea N, Molinero O, Martinez-Garcia R, Jimenez-Jimenez R, Gonzalez- Gallego J, Marquez S, Feeling of wellbeing in elderly people: relationship to physical activity and physical Function, Archives of Gerontology and Geriatrics, (2009), Vol.48 No.1, pp.306-312.

[3] Takata Y, Ansai T, Soh I, Awano S, Yoshitake Y, Kimura Y, Sonoki K, Kagiyama S, Yoshida A, Nakamichi I, Hamasaki T, Torisu T, Toyoshima K, Takehara, T, Quality of life and physical fitness in an 85-year-old population, Archives of Gerontology and Geriatrics, (2010), Vol.50 No.1, pp.272-276.

[4] S. Lim, S. Chang, Nursing home staff members' subjective frames of reference on residents' achievement of ego integrity: a q-methodology study, Japan Journal of Nursing Science, (2018), Vol.15 No.1, pp.17-30.

[5] S. Lim, S. Chang, Ego-integrity management of residents in nursing homes: a concept analysis based on the method by Walker and Avant, Journal of Korean Gerontological Nursing, (2018), Vol.20 No.2, pp.97-108.

[6] E. H. Erickson, Childhood and Society, W.W. Norton \& Company, (1963)

[7] E. H. Erickson, Identity and the life cycle, W.W. Norton \& Company, (1980)

[8] E. H. Erickson, The life cycle completed, W.W. Norton \& Company, (1982)

[9] S. Lim, S. Chang, S. Kim, H. Kim, J. Choi, M. Park, Nurses' management of nursing home residents' remaining functional ability: concept development, Journal of Korean Academy of Fundamentals of Nursing, (2014), Vol.21, No.1, pp.57-68.

[10] M. Sandelowski, Whatever happened to qualitative description?, Research in Nursing \& Health, (2000), Vol.23 No.4, pp.334-340.

[11] K. Krippendorff, Content analysis: an introduction to its methodology, SAGE publication, (2018)

[12] H. Hsieh, S. Shannon, Three approaches to qualitative content analysis, Qualitative Health Research, (2005), Vol.15, No.9, pp.1277-1288.

[13] B. Wadensten, Changes in nursing home residents during an innovation based on the theory of gerotranscendence, International Journal of Older People Nursing, (2010), Vol.5, No.2, pp.108-115.

[14] Ali WGM, Ayoub NS, Nurses' attitudes toward caring for dying patient in Mansoura university hospitals, Journal of Medicine and Biomedical Sciences, (2010), Vol.3, No.1, pp.16-23. ISSN 2078-0273

[15]S. Park, Y. Hwang, Death Awareness and Death Preparation Experiences in Hospitalized Elderly People in a LongTerm Care Facility, Journal of Elderly Welfare Promotion and Management, (2017), Vol.1, No.1, pp.21-28.

[16] I. Byock, The nature of suffering and of opportunity at the end of life, Clinics in Geriatric Medicine, (1996), Vol.12, No.2, pp.237-252.

[17] S. Aleksandrova-Yankulovska, H. Have, Survey of Staff and Family Members of Patients in Bulgarian Hospices on the Concept of 'Good Death', American Journal of Hospice and Palliative Care, (2013), Vol.32, No.2, pp.226-232.

[18] S. Kim, M. Kim, H. Kim, J. Choi, S. Chang, Nursing home nurses' ways of knowing about peaceful deaths in endof-life care of residents: personal knowledge and strategies, Journal of Hospice \& Palliative Nursing, (2014), Vol.16, No.7, pp.438-445.

[19]H. Hyun, E. Choi, M. Byeon, Jin. Kim, The Efficacy of Dementia Prevention Integrative Program for Cognitive function, Depression, and Quality of life in the Elderly Non-Dementia, Journal of Elderly Welfare Promotion and Management, (2017), Vol.1, No.1, pp.43-50.

[20]Chung Cho, Sook Han, The Effect of Reminiscence Intervention on the Elderly Population: a Systematic Review, International Journal of Elderly Welfare Promotion and management, (2018), Vol.2, No.1, pp.33-38. 